Michael Herslund*

\title{
Argumentation et cohésion textuelle : l'exemple de en plein 1
}

Une partie importante du contenu de certains éléments linguistiques est constituée par leur orientation argumentative, voir par exemple Ducrot (1980), Anscombre et Ducrot (1983), Lundquist (1987). Il s'ensuit que le choix et la combinaison de certains lexèmes et de certaines expressions, à l'exclusion de certains autres qui auraient été parfaitement possibles dans le même contexte, traduisent et correspondent à une certaine visée argumentative. La fonction textuelle de certains éléments, qui a priori ne semblent avoir rien à voir avec l'argumentation, est donc d'amener le destinataire à tirer certaines conclusions. Cette fonction est illustrée de façon particulièrement éloquente par la locution prépositionnelle en plein. Je parle de "locution prépositionnelle" par pure commodité. On pourrait en effet discuter s'il ne s'agit pas plutôt de la préposition en suivi d'un régime comportant l'épithète plein, mais cette discussion n'est pas pertinente pour mon propos ici.

Rien ne semble prédestiner en plein à jouer un rôle dans l'argumentation. Si on consulte les dictionnaires, on apprend, par le Petit Robert, que en plein suivi d'un substantif veut dire "au milieu de (espace)". Le Grand Robert dit à peu près la même chose, mais reconnaît en outre "une valeur intensive" à l'expression. Mais c'est à peu près tout : dictionnaires et grammaires s'accordent pour attribuer à en plein le sens "géométrique" de 'au milieu de' ou le sens "intensif" de 'remplissage complet' (cf. Togeby 1984:151), sens qui ne conviennent pour ainsi dire jamais à l'expression : le sens retenu comme fondamental, 'au mi-

1 Je remercie Irène Baron pour ses remarques judicieuses et sur le fond et sur la forme de cet article.

* Michael Herslund

Handelshфjskolen i København

Institut for fransk

Dalgas Have 15

DK-2000 Frederiksberg 
lieu de', ne semble pas du tout rendre compte de notre tour puisque en pleine rue n'est pas synonyme de au milieu de la rue (au sens géométrique) et en plein $X X^{e}$ siècle ne veut surtout pas dire la même chose que en 1950. Notons aussi que en plein a aussi et, semble-t-il, surtout, un sens temporel.

Ce qu'on remarque quand on regarde de plus près la fonction textuelle de en plein c'est que cette expression introduit un élément qui peut traduire la simple surprise devant l'insolite ou le scandale causé par l'inouï. Dans certains cas, en plein a pour fonction essentielle de souligner l'horreur ou le scandale d'un événement :

(1) Un lycéen de treize ans touché en pleine tête (Nouvel Observateur sept. 1978, 53)

Le 747 piraté d'Indian Airlines vient d'exploser en plein vol, au-dessus du Channel (Express 24.2.89, 24)

Nous retrouvons là le "sens intensif" du Grand Robert. Mais on voit tout de même un effet argumentatif en germe. Seulement, dans les exemples de (1), cette argumentation fait appel aux sentiments du destinataire plutôt qu'à une analyse logique. Et souvent aussi, l'effet n'est pas une valeur argumentative à proprement parler, mais plutôt un effet cocasse :

(2) L'idée de courir immédiatement à Berlin, en troisième classe, bien entendu, pour tuer Hitler en pleine canicule, avec tout ce que cela supposait d'énervement, de fatigue et de préparatifs, ne me souriait guère (Gary Promesse 215)

Je vois souvent des Français faire des prodiges d'équilibre et d'acrobatie en plein milieu d'un boulevard sillonné de voitures (Daninos Carnets 50)

Mais le plus souvent, la locution en plein introduit entre deux éléments un contraste, dont la fonction est clairement d'appuyer l'argumentation du texte. Prenons comme point de départ l'exemple suivant avec le sens temporel de en plein:

(3) En plein siècle des lumières, le jésuite Athanase Kircher affirmait que les hiéroglyphes resteraient à jamais indéchiffrables. Imprudent! (Express 7.8.87, 10)

L'argumentation inhérente à ce morceau de texte, signalée justement par en plein au lieu du simple $a u$, est révélée par la paraphrase suivante qu'on peut présenter sous forme de topos (cf. Ducrot 1980, Anscombre et Ducrot 1983) : 
(3') Si, en général, on n'affirme pas avec trop d'assurance que telle chose est impossible, à plus forte raison on ne le fait pas au siècle des lumières où on commence à croire au progrès et aux possibilités illimitées de l'esprit humain.

Dans (3), le contraste introduit est donc le contraste entre les affirmations de Kircher et son époque. La conclusion visée, traduite aussi par le Imprudent! final, est évidemment que de telles affirmations détonnent au siècle des lumières peu de temps avant la découverte de Champollion qui devait les contredire.

L'exemple suivant montre l'emploi local de notre expression :

... deux journalistes (...) ont été arrêtés pour avoir organisé en

plein Rome une parodie de discours pontifical (Nouvel Observateur $30.10 .78,48$ )

Notons d'abord que en plein ne véhicule pas du tout une information de caractère topographique : ce n'est pas l'endroit exact du happening qui importe. Ici, comme ci-dessus, on peut construire une paraphrase, sous forme de topos, qui dégage l'argumentation :

(4') Si, en général, on ne doit pas parodier les grands de ce monde, il faut une audace singulière pour parodier le pape à Rome.

De nouveau, une telle paraphrase est autorisée par en plein. Si on enlève cette expression des deux exemples qu'on vient de voir, d'argumentatifs les textes deviennent banalement factuels. Et si, dans l'exemple (4), on substitue à Rome un autre nom de ville tel que Téhéran ou Nogentle-Rotrou, on a du mal à reconstruire le sens argumentatif - Deux journalistes ont été arrêtés pour avoir organisé en plein Nogent-le-Rotrou une parodie de discours pontifical - qui repose justement sur le contraste entre Rome et parodie de discours pontifical. Ce qui a pour conséquence que en plein devient un signal important au destinataire. La locution invite celui-ci à trouver le contraste et l'effet argumentatif qui en découle et qui justifie l'emploi de la locution. Prenons un troisième exemple :

L'assassinat de Marat le 13 juillet 1793, concrétisa l'immensité du péril : en plein Paris révolutionnaire, Charlotte Corday, une jeune royaliste de Normandie, avait pu tuer l'Ami du peuple (Soboul Revolution 303)

De nouveau, il est clair que en plein Paris n'est pas une simple indication topographique. De nouveau, une paraphrase topique est facilement imaginable. Mais il est clair aussi que pour quelqu'un qui ignore 
tout de la révolution française, de Marat et de Charlotte Corday, l'exemple (5) ne véhicule rien de spécial, à part le fait que le texte raconte l'assassinat de quelqu'un qui s'appelle Marat par quelqu' un qui est qualifié de royaliste. Or, c'est là que la fonction de en plein devient cruciale : la locution invite le lecteur à trouver l'aspect remarquable de cette affaire. Le syntagme en plein Paris révolutionnaire permet au destinataire qui ne connaît pas l'histoire, ou la connaît médiocrement, de la reconstituer, dans ses grandes lignes au moins. Et plus particulièrement en ce qui concerne le rapport entre Marat et le mystérieux Ami du peuple : l'expression en plein, en soulignant l'opposition entre Paris révolutionnaire et une jeune royaliste, permet au destinataire non averti de conclure que Marat - l'Ami du peuple désigne probablement un leader révolutionnaire, plutôt qu'un amant volage ou un commerçant frauduleux. Or, que les royalistes tuent les révolutionnaires, et vice versa, cela n'a rien de bien remarquable. Ce qui est remarquable en revanche, c'est que l'assassinat a pu avoir lieu à Paris, le haut-lieu de la révolution.

Précisons maintenant le contraste introduit par en plein. Il s'agit d'un contraste lexical entre le syntagme en plein, qui a le plus souvent la fonction de syntagme adverbial de temps ou de lieu, et le prédicat de la proposition où se trouve ce complément adverbial. Dans la réplique de film suivant - on se trouve dans un grand jardin devant un manoir magnifique - le texte est pour ainsi dire réduit à ces deux éléments (et le prédicat, à son tour, est réduit à un complément de lieu) :

(6) A la campagne - en plein faubourg de Paris (du film Camille Claudel)

De même dans les exemples de notre expression qui vont suivre, où on voit cet effet de contraste :

... enfin inauguration d'un pont de lianes audacieusement jeté par-dessus un ravin de cent pieds de profondeur en pleine forêt tropicale (Tournier Vendredi 92)

Quand il rencontrait Léopold sur son passage, il se contentait de lui donner un coup de pied sans dire un mot. Un vrai cosaque en pleine zone résidentielle (Fleischman Rendez-vous 58)

La demeure de Rondelet était sise en la rue Bout-du-Monde, étrange nom pour une rue en plein mitan de la ville (Merle Vertes années 185) 
... cette autre (...) se faisait mitrailler en pleine rue, la chargeait de codes secrets tandis qu'elle se faisait agresser en plein jour par un moustachu (Deforges Tango 172)

c'était comme une besogne de nuit faite en plein jour, par un travailleur morne, puissant et muet (Zola Assomoir 50)

Salman Rushdie : Soyons sérieux! Il faut être complètement parano pour s'imaginer, en plein $\mathbf{X} \boldsymbol{X}^{\boldsymbol{e}}$ siècle, qu'une religion aussi ancienne que l'islam puisse être menacée par un livre (Express 3.3.89, 10)

Faites la connaissance d'un pays qui continue à construire des cathédrales en plein $\boldsymbol{X} \boldsymbol{X}^{\boldsymbol{e}}$ siècle (Express 27.1.89,2)

Mais le "transi" de Ligier Richier (un squelette élevant son coeur vers le ciel) perpétue en pleine Renaissance la tradition médiévale de la danse macabre (Lagarde et Michard XVI siècle 9)

En plein drame du chômage, 39000 emplois ne sont pas pourvus (Express 22.2.85, 23)

Les citoyens, en pleine guerre, arrivent à Bordeaux à pied plus vite que les Allemands en blindés (Daninos Nouveaux carnets 108)

mais Bénigne Bossuet lui-même a été obligé de faire allusion à une poule en pleine oraison funèbre, et il s'en est tiré avec pompe (Hugo Misérables 359)

Des professeurs connus pour leur attachement à la laïcité sont attaqués en plein cours par des commandos de "barbus" (Evénement 4-10.1.90, 59)

C'est en plein succès, au terme d'un parcours qu'il juge luimême parachevé, qu'il va se donner la mort (Bona Gary 395)

Bien avant d'atteindre l'âge de la retraite, on devient maintenant grand-père ou grand-mère en pleine période d'activité professionnelle (Andersen \& Guillois Hexagone 58)

M. Daninos (...) me dit un jour, en pleine retraite des Flandres, combien il regrettait de se trouver au coeur de la bataille (Daninos Carnets 61)

On voit, dans tous les cas, comment la fonction de en plein est d'inviter le destinataire à identifier un contraste et à tirer des conclusions à partir de ce contraste. 
Si en plein signale la présence d'une intention argumentative, on peut s'attendre à trouver cette expression accompagnée du morphème même qui, comme démontré par Ducrot (1980) et Nølke (1983), introduit justement un argument plus fort en vue d'une certaine conclusion. Les exemples suivants illustrent cette combinaison :

(8) Au fond, la forge luisait, même en plein midi (Zola Assomoir 215)

Les rideaux étaient toujours baissés; même en plein jour, la lampe était allumée (Gary Vestiaire 163)

Seul, naguère avec ses deux femmes, dans le plaisir ou la querelle avec la première, dans une tendresse parfois douce-amère avec l'autre (il est trop sincère pour envisager leurs relations différemment, même en plein deuil) (Yourcenar Quoi? 11)

Un endroit désolé, consumé de soleil, calciné même en plein hiver, pareil, pour la couleur et le désordre, à un vaste foyer dont il ne resterait plus que des cendres (Fromentin, cit. Grand Robert 2.278)

en cette saison de déréliction souveraine où l'on ne voyait plus ses propres mains dans les rues sous les réverbères qui restaient allumés même en plein midi (Butor Emploi 236)

Dans tous ces exemples, on voit facilement le même effet argumentatif que plus haut. Dans tous les cas, l'argumentation passe par un topos qu'on pourrait formuler comme (8') :

(8') A l'époque ou à l'endroit désignés par le syntagme en plein, il ne se passe pas normalement/il ne doit normalement pas se passer ce que désigne le prédicat.

Et c'est sur la partie 'normalement' de ce topos que se greffe le morphème même pour souligner l'effet argumentatif.

Regardons maintenant, pour conclure cette petite esquisse, le rôle que joue, dans l'intégration textuelle des énoncés, c'est-à-dire dans la cohésion textuelle, la valeur argumentative du tour. Et prenons comme point de départ l'exemple suivant :

(9) Un plan d'aide économique, un plan Marshall pour la Pologne? En plein centre de Varsovie, le chantier du métro n'en finit pas de finir (Parisien 21.9.89, 4)

Quel est le rapport entre les deux énoncés qui constituent ce petit texte? Le premier pose la question sur l'opportunité d'un plan Marshall pour 
la Pologne et le second apporte un argument contre un tel plan en renvoyant à un topos tel que (9') :

(9') Si à Varsovie, la capitale, on n'arrive pas à terminer les travaux entrepris, cela va sûrement encore plus lentement ailleurs.

Le destinataire n'a probablement pas trop de peine à tirer la conclusion souhaitée : non, ce n'est pas la peine. Et c'est la présence de en plein qui fait reconstituer au destinataire ce topos, et la conclusion qui en suit. Autrement dit, la cohésion est fortement appuyée par cette locution.

L'exemple suivant montre comment l'emploi d'un mot, en l'occurrence le mot litote est justifié par le contexte suivant à travers le contraste introduit par en plein :

(10) Le ministre des Affaires étrangères s'efface parfois (...) devant le grand avocat d'affaires qu'il fut (...) il s'est, cette fois, surpassé dans l'art de la litote. En pleine année du bicentenaire de la Révolution française, le chef de la diplomatie française s'est déclaré "choqué” de la condamnation à mort par Khomeini de l'auteur des "Versets sataniques". (-) Vous avez dit choqué, monsieur le Ministre? (Express 3.3.89, 6)

Le mot litote est expliqué par la suite : si tout le monde est choqué par la condamnation de Rushdie, on attend du ministre des affaires étrangères français des déclarations autrement violentes en 1989, année où on fête la liberté et les droits de l'homme.

L'apparition du mot défi dans le texte suivant est motivée également par un contraste souligné par en plein :

(11) Comment commémorer un événement aussi tellurique, et aux multiples retombées, en plein été? Tel était le défi qu'il fallait relever (Libération 12.7.89)

Ce qui constitue un défi, et ce qui autorise l'emploi du mot défi au lieu de problème, question, etc., c'est la résolution de la contradiction introduite et soulignée par en plein entre commémorer (à savoir la Révolution française) et été (époque où les Français sont en vacances), couple de mots qui, par ailleurs, ne constitue pas une contradiction.

Le dernier exemple est l'exemple (5) déjà cité, qui sera répété ici :

L'assassinat de Marat le 13 juillet 1793, concrétisa l'immensité du péril : en plein Paris révolutionnaire, Charlotte Corday, une jeune royaliste de Normandie, avait pu tuer l'Ami du peuple (Soboul Revolution 303) 
Qu'est-ce qui démontre en effet l'immensité du péril mieux que l'assassinat de Marat, en plein Paris révolutionnaire? Même à Paris, le hautlieu de la révolution, les révolutionnaires ne sont pas en sécurité. Et c'est le contraste introduit et souligné par en plein entre Paris révolutionnaire et tuer l'Ami du peuple qui autorise la conclusion : le péril était immense, et qui justifie le choix de mots du premier énoncé.

On voit donc que la locution en plein n'est ni une expression à valeur géométrique, ni un élément d'intensité “innocent". Son emploi souligne le plus souvent un contraste ou une contradiction, et la locution autorise, en renvoyant à un topos qui joue sur une telle contradiction, une conclusion qui, si elle est explicitée, ce qui est loin d'être toujours le cas, contribue à son tour à établir la cohésion textuelle.

\section{Bibliographie}

Anscombre, Jean-Claude \& Oswald Ducrot (1983) : L'argumentation dans la langue. Bruxelles : Mardaga.

Ducrot, Oswald (1980) : Les échelles argumentatives. Paris : Minuit.

Lundquist, Lita (1987) : Programme argumentatif et désambiguïsation référentielle. In : Revue Romane 22.163-181.

Nølke, Henning (1983) : Les adverbes paradigmatisants. Fonction et analyse. Etudes Romanes de l'Université de Copenhague 23. Copenhague : Akademisk Forlag.

Togeby, Knud (1984) : Grammaire française. Volume IV : Les mots invariables. Edité par Magnus Berg, Ghani Merad \& Ebbe Spang-Hanssen. Etudes Romanes de l’Université de Copenhague. Copenhague : Akademisk Forlag.

\section{Textes cités}

Andersen, F. \& N. Guillois : Aux quatre coins de l'Hexagone. Samfundslitteratur.

Bona, Dominique : Romain Gary. Mercure de France.

Butor, Michel : L'emploi du temps. Minuit.

Daninos, Pierre : Les carnets du major Thompson. Livre de poche.

Daninos, Pierre : Nouveaux carnets du major Thompson. Livre de poche.

Deforges, Régine : Noir tango. Livre de poche.

L'événement du jeudi.

L'Express.

Fleischman, Cyrille : Rendez-vous au métro Saint-Paul. Le Dilettante.

Gary, Romain : La promesse de l'aube. Folio.

Gary, Romain : Le grand vestiaire. Folio. 
Hugo, Victor : Les misérables. Folio.

Lagarde, A. \& L. Michard : XVI $I^{e}$ siècle. Bordas.

Libération.

Merle, Robert : En nos vertes années. Presses-Pocket.

Le Nouvel Observateur.

Le Parisien.

Soboul, Albert : La révolution française. Gallimard.

Tournier, Michel : Vendredi ou les limbes du Pacifique. Folio.

Yourcenar, Marguerite : Quoi? L'éternité. Folio.

Zola, Emile : L'assomoir. Livre de poche. 